\title{
Professor Klaus Kirchgässner
}

In early 2011, Bernold Fiedler, Mariana Haragus, and Alexander Mielke had joined with Geneviève Raugel and Yingfei Yi as Special Editors of a future special volume celebrating the 80th birthday of Klaus Kirchgässner.

Klaus Kirchgässner did not reach his 80th birthday. On Saturday July 9, 2011, we lost our dear friend and colleague. This birthday special volume became a volume to his memory. It will appear in the combined September-December 2015 issue of the Journal.

The five special Editors of the Klaus Kirchgässner volume share, with the authors of the papers in this Volume and with his many friends, a deep sadness over his loss. We will all remember him for ever as an excellent scientist with deep mathematical insight and high standards, as a devoted teacher and colleague and as a friendly and caring person with a wonderful sense of humor.

We thank Bernold Fiedler, Mariana Haragus, and Alexander Mielke for their valuable help.

Professor George R. Sell, co-editor in chief of the Journal of Dynamics and Differential Equations, sadly passed away on May 29, 2015. He tirelessly gave us help and support during the editorial preparation of this Volume.

Geneviève Raugel and Yingfei Yi

Co-Editors in Chief

Geneviève Raugel

CNRS, Laboratoire de Mathématiques, Univ. Paris-Sud, Orsay, France

e-mail: genevieve.raugel@math.u-psud.fr

Yingfei Yi

University of Alberta, Edmonton, Canada

Georgia Institute of Technology, Atlanta, GA, USA

e-mail: yingfei@ualberta.ca, yi@math.gatech.edu

Received: 10 September 2015 / Published online: 23 October 2015

(C) Springer Science+Business Media New York 2015 


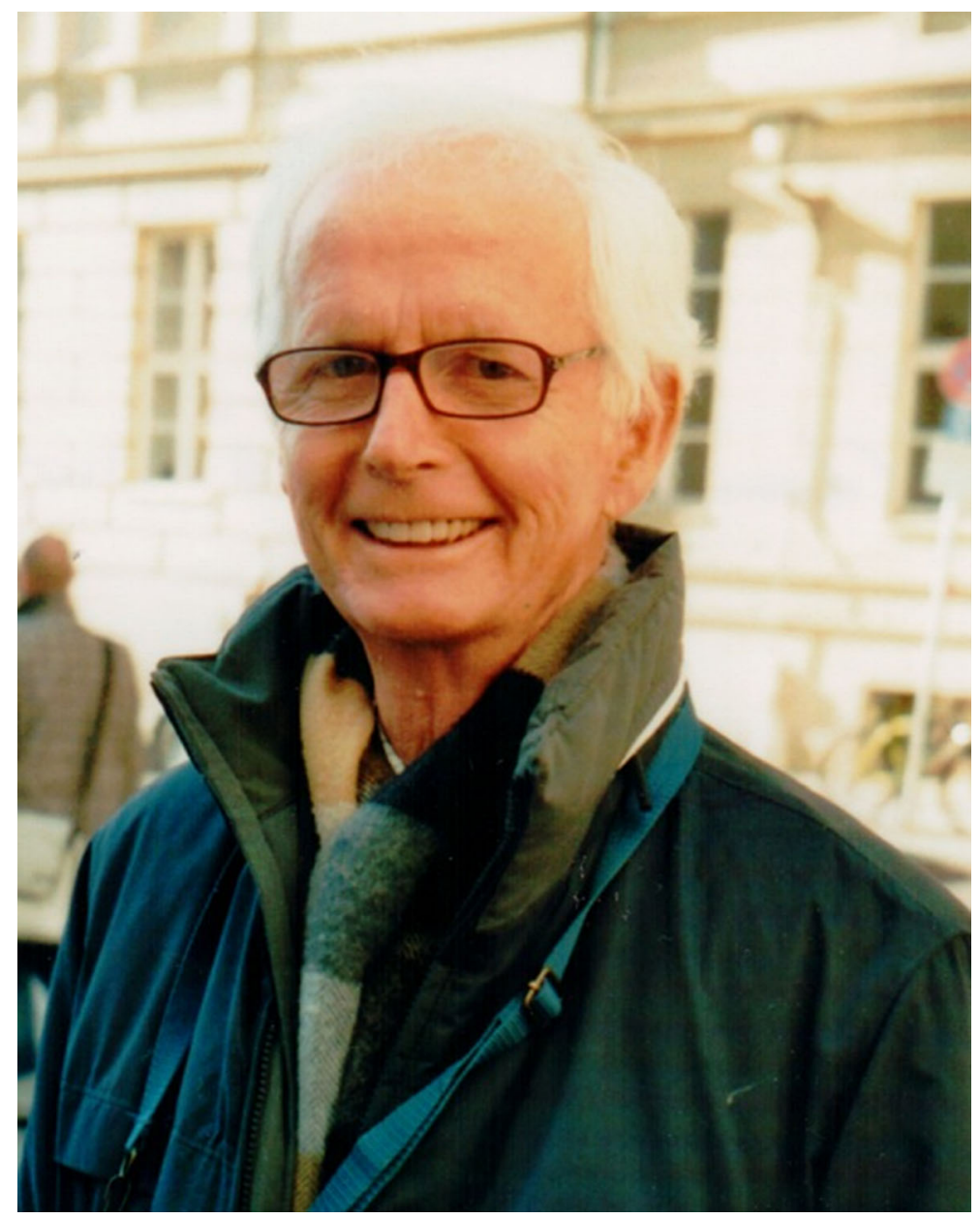

Professor Klaus Kirchgässner 